\title{
Extension of quantum molecular dynamics for production of light complex particles in nucleon-induced reactions
}

\author{
Y. Watanabe ${ }^{1, a}$ and D.N. Kadrev ${ }^{1,2}$ \\ 1 Department of Advanced Energy Engineering Science, Kyushu University, Kasuga, Fukuoka 816-8580, Japan \\ 2 Institute for Nuclear Research and Nuclear Energy, Bulgarian Academy of Sciences, Sofia 1784, Bulgaria
}

\begin{abstract}
A quantum molecular dynamics (QMD) model is applied to production of light charged particles in nucleon-induced reactions on several light and medium heavy nuclei. The generalized evaporation model (GEM) is used to deal with the statistical decay process of highly excited fragments at the end of the QMD stage. Good agreement with experimental double-differential cross sections is obtained for nucleon emission, but the calculation shows remarkable underprediction for preequilibrium emission of light complex particles, i.e., d, t, ${ }^{3} \mathrm{He}$, and ${ }^{4} \mathrm{He}$. To improve the situation, a phenomenological surface coalescence model is incorporated into the QMD simulation under the assumption that light complex particles are mainly formed near the surface region by a leading nucleon that is ready to escape from the nucleus during the dynamical process.
\end{abstract}

\section{Introduction}

High-energy nucleon-induced reactions take place via two major reaction processes, namely dynamical process (e.g., spallation and preequilibrium processes) and statistical decay process. The intra-nuclear cascade (INC) model [1,2] and preequilibrium model [3] are the typical models to describe the dynamical process which occurs during a short reaction time just after the interaction of an incident nucleon with a target nucleus. These models are incorporated into the high-energy transport codes that are widely used in various accelerator applications, space development, etc.

A quantum molecular dynamics (QMD) model [4,5] is available as an alternative model to describe the dynamical processes in nuclear reactions. The QMD has been developed as one of the microscopic simulation tools that are used in heavy-ion nuclear physics. The applicability to nucleoninduced reactions has also been demonstrated and experimental double-differential nucleon emission cross sections can be reproduced well [5-7]. The JQMD code [5] was partly used in the nuclear data evaluation of JENDL highenergy file for energies from $150 \mathrm{MeV}$ up to $3 \mathrm{GeV}[8,9]$. Moreover, it has recently been incorporated into Monte Carlo particle transport calculations for applications (e.g., PHITS code [10]). However, recent comparisons with experimental data have revealed remarkable underprediction in nucleoninduced preequilibrium emission of light complex particles $\left(\mathrm{d}, \mathrm{t},{ }^{3} \mathrm{He}\right.$, and $\left.{ }^{4} \mathrm{He}\right)$, which are hereafter abbreviated as LCPs.

From the viewpoint of applications, a reliable prediction of the production yields of hydrogen and helium isotopes is particularly important for damage assessment in materials relevant to design of accelerator-driven systems and spallation neutron sources. Therefore, it is required to improve the underprediction seen in preequilibrium emission of LCPs to apply the QMD to such applications. It has recently been demonstrated that the preequilibrium LCP emission can be described reasonably well by implementation of a surface

${ }^{a}$ Presenting author, e-mail: watanabe@aees . kyushu-u.ac .jp coalescence mechanism in the INC model for nucleon-induced reactions at several hundreds of $\mathrm{MeV}$ to $2.5 \mathrm{GeV}[11,12]$. In the past, a similar coalescence idea was taken into account in the preequilibrium exciton model, and successfully applied to light-ion production in proton-induced reactions at incident energies below $100 \mathrm{MeV}$ [13-15]. In the present work, we also implement such a phenomenological surface coalescence model into the QMD simulation [16]. The extended QMD is used to analyses of some experimental data of neutron-induced light-ion emission from $\mathrm{O}, \mathrm{Si}$, and $\mathrm{Fe}$ at $E_{n}=96 \mathrm{MeV}$ [17-19] and $\mathrm{Cu}$ at $542 \mathrm{MeV}$ [20].

\section{Model}

\subsection{Quantum molecular dynamics}

The QMD method is a semiclassical simulation method to deal with the time evolution of nucleon many-body system. Each nucleon propagates in nuclear mean field formed by all other nucleons in a self-consistent way. When two nucleons come close, it is assumed that they suffer from a stochastic two-body collision. We use the JQMD code [5] in the present work. The QMD model used in the code is outlined below.

The total wave function is assumed to be a direct product of single-particle wave function which is expressed by a Gaussian wave packet of width $L$ :

$$
\phi_{i}(\mathbf{r})=\frac{1}{(2 \pi L)^{3 / 4}} \exp \left[-\frac{\left(\mathbf{r}-\mathbf{R}_{i}\right)^{2}}{4 L}+\frac{i}{\hbar} \mathbf{r} \cdot \mathbf{P}_{i}\right],
$$

where the variables $\mathbf{R}_{i}$ and $\mathbf{P}_{i}$ are the centers of position and momentum of the $i$ th nucleon, respectively. The time evolution of $\mathbf{R}_{i}$ and $\mathbf{P}_{i}$ is described by the following Newtonian equations of motion:

$$
\dot{\mathbf{R}}_{i}=\frac{\partial H}{\partial \mathbf{P}_{i}}, \quad \dot{\mathbf{P}}_{i}=-\frac{\partial H}{\partial \mathbf{R}_{i}},
$$


where $H$ denotes the total Hamiltonian consisting of the kinetic energy, two- and three-body Skyrme type interactions, the Coulomb and the symmetry terms. In addition, twobody collision process is considered in a phenomenological way. Namely, if two nucleons come closer and their impact parameter becomes smaller than a given value, $\left(\sigma_{N N} / \pi\right)^{1 / 2}$, determined from the $N N$ scattering cross section, $\sigma_{N N}$, these nucleons are scattered. In the collision process, the Pauli blocking effect in the final state is taken into account.

The QMD simulation continues up to a certain time (e.g., $100 \mathrm{fm} / \mathrm{c}$ in the present work) after a nucleon incidence on the target nucleus. The generated LCPs and fragments are identified at the end of the QMD simulation. The JQMD code adopts the phase-space cluster chain method [21] as the identification procedure, i.e., if two nucleons are within a certain distance ( $4 \mathrm{fm}$ as the default value) in the coordinate space and their relative momentum is less than the local Fermi momentum, then they are judged to be bound in the same cluster. Particle emission from the thermally equilibrated residual system is considered as statistical decay, and the GEM code based on the generalized evaporation model [22] is used.

\subsection{Phenomenological surface coalescence model}

Some modifications [16] have been made in the QMD calculation on the basis of the phenomenological prescriptions incorporated in the INC model by Letourneau et al. [11] and Boudard et al. [12], and the coalescence exciton model by Iwamoto et al. [13,14] and Bisplinghoff [15].

We assume that a leading nucleon which is ready to leave the composite nucleus, i.e., the reaction system consisting of the incident nucleon and the target nucleus, can be involved in the formation of LCPs in the nuclear surface where the nucleon density is relatively low. Each time one of fast nucleons generated by a series of two-body collisions during the QMD stage passes the border defined by a distance $D$ outside the sphere of radius $R_{0}$, it is regarded as the leading nucleon and then the following test for formation and emission of LCPs is implemented.

It is assumed that LCPs are formed by successive coalescence starting from the leading nucleon. Namely, the candidate LCP is chosen by inspecting whether or not the following phase space condition $[11,12]$ is satisfied:

$$
R_{i m} \times P_{i m} \leq h_{0},
$$

where $R_{i m}$ and $P_{i m}$ are the relative spatial and momentum coordinates of the considered $i$ th nucleon with respect to the intermediate cluster $m$. In the first stage of deuteron formation, a counterpart nucleon of the $i$ th nucleon corresponds to the intermediate cluster $m$ in equation (3). The value $h_{0}$ is an adjustable parameter. We impose an additional constraint that the center of mass of the cluster should be located far from the radius $R_{0}$, in order to ensure that the candidate cluster is formed in the surface layer region. In the present work, the formation of four kinds of LCPs $\left(\mathrm{d}, \mathrm{t},{ }^{3} \mathrm{He}\right.$, and $\left.{ }^{4} \mathrm{He}\right)$ is considered.

Finally, we check whether or not the candidate LCP can be emitted with respect to the kinetic energy and the Coulomb barrier penetration, according to the order of the same priority list, ${ }^{4} \mathrm{He}>\left({ }^{3} \mathrm{He}\right.$ or $\left.\mathrm{t}\right)>\mathrm{d}$, as in ref. $[11,12]$. The kinetic energy of the LCP denoted by $\varepsilon_{c}$ can be calculated as below and it should be positive for the emission:

$$
\varepsilon_{c}=\sum_{j=1}^{A_{c}}\left(E_{j}+V_{j}\right)+B_{c},
$$

where $E_{j}$ and $V_{j}$ are the kinetic energy and the potential energy of the $j$ th constituent nucleon, respectively, and $A_{c}$ and $B_{c}$ are the mass number and the binding energy of the LCP, respectively.

If the test for emission fails for all LCP candidates, the leading nucleon under consideration is emitted. Then the timeevolution of the system proceeds until the next leading nucleon appears, and the same procedure of the LCP formation is repeated again as mentioned above. This algorithm is continued until the end of the QMD stage and is finally switched to the statistical decay calculation.

\section{Results and discussion}

The extended QMD model was first applied to light-ion production from neutron-induced reactions on $\mathrm{O}, \mathrm{Si}$, and $\mathrm{Fe}$ at $E_{n}=96 \mathrm{MeV}$ [16]. The QMD simulation was performed with total $10^{6}$ events for the major isotopes ${ }^{16} \mathrm{O},{ }^{28} \mathrm{Si}$ and ${ }^{56} \mathrm{Fe}$. The angular and energy distributions of emitted light ions were averaged and converted finally to the double-differential cross sections using the geometrical cross section, $\pi b_{\text {max }}^{2}$, where $b_{\max }$ is the maximum impact parameter. The $b_{\max }$ value was determined so that the calculated reaction cross section was saturated: $b_{\max }=5.0,5.5$, and $7 \mathrm{fm}$ for ${ }^{16} \mathrm{O},{ }^{28} \mathrm{Si}$ and ${ }^{56} \mathrm{Fe}$, respectively. For the Gaussian width in equation (1), $L=$ $2.0 \mathrm{fm}^{2}$ was used. We adopted the default values used in the JQMD code for the other parameters, e.g., the parameters contained in the effective interaction.

Some adjustable parameters are included in the surface coalescence model mentioned above. For the radius $R_{0}$, the rms radius of the ground state was chosen. Since the distance $D$ was found to be sensitive to the rms-half distance of two nucleons in the formed deuteron, the value was finally determined as $2.3 \mathrm{fm}$ to reproduce the experimental value [23]. The value of $h_{0}$ was fixed to be $260 \mathrm{MeV} \mathrm{fm} / \mathrm{c}$ so that a reasonable fit to the experimental data was obtained as shown below.

Figures 1 and 2 show the angle-integrated energy spectra of light-ion production for $\mathrm{Si}$ and $\mathrm{O}$ in comparison with the experimental data $[17,18]$. Our new results present the enhancement of production of LCPs in the medium emission energy range compared to the original QMD calculation, and agreement with the experimental data is fairly improved. However, the improved QMD calculation still exhibits large underestimation around the high energy end of the emission spectra. Note that the same is true for Fe. The underestimation may be partly due to the other reaction processes, such as the direct pick-up process by the incident neutron and the knockout process of preformed LCPs, which cannot be described properly within the present QMD framework. Particularly, 


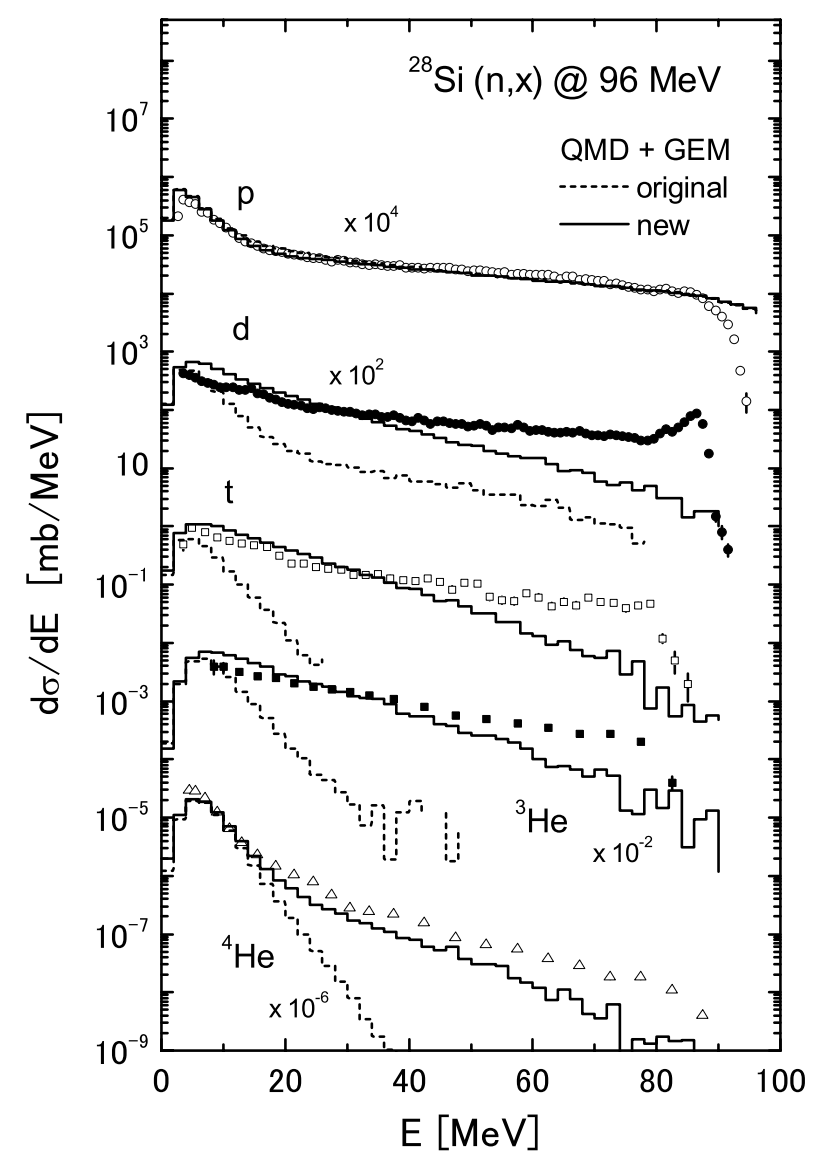

Fig. 1. Angle-integrated energy spectra of all light ions produced from the reaction $\mathrm{n}+\mathrm{Si}$ at $96 \mathrm{MeV}$. The experimental data are taken from ref. [18].

the large bump observed around the high energy end of the deuteron spectra corresponds to the transition to the ground state and low-lying excited states of the residual nucleus via direct pickup of a proton. It is expected that this region may be described well by a quantum-mechanical calculation based on DWBA.

In figure 2 , the sensitivity of the radius parameter $R_{0}$ to production of LCPs is examined. The value of $3.2 \mathrm{fm}$ corresponds to the rms radius of the ground state by the QMD calculation. The yields increase for use of the smaller $R_{0}$, and the effect is more appreciable as the LCP becomes heavier. This enhancement may be reasonably explained from the fact that number of nucleons participated in the formation of LCPs increases when the smaller $R_{0}$ is used.

Next, similar calculations are extended to nucleon-induced reactions at higher incident energies. Figure 3 shows a comparison between the calculated double-differential cross sections for neutron-induced production of protons, deuterons, and tritons on $\mathrm{Cu}$ at $542 \mathrm{MeV}$ and the experimental data [20]. In the calculation, the parameter $h_{0}$ is set to be $330 \mathrm{MeV} \mathrm{fm} / \mathrm{c}$ so as to obtain better agreement with the experimental data. Enhancement of LCP production in the QMD calculation that takes account of the surface coalescence of LCPs results in fairly good agreement with the measurement, although underestimation is seen at forward angles for deuteron production

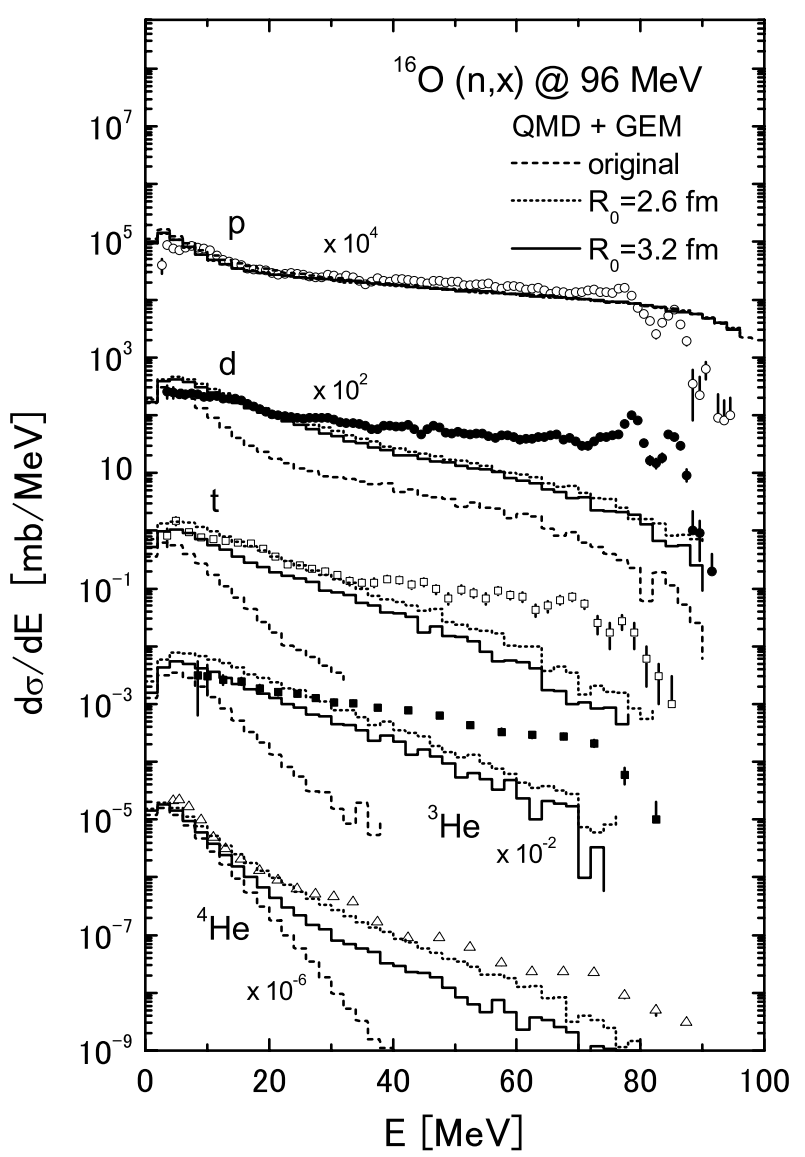

Fig. 2. Same as in figure 1 , but the reaction $n+O$ at $96 \mathrm{MeV}$. Two results calculated with different $R_{0}$ values are plotted. The experimental data are taken from ref. [17].

and overestimation at backward angles for triton production. Reduction of proton production yield is obviously seen in the extended QMD calculation compared with the original QMD calculation, particularily at backward angles, whereas the higher energy component emitted in the forward direction does not change. As a result, the agreement with the measurement is improved for all emission angles. Similar reduction is seen in the INC calculation implementing the surface coalescence method for the reaction $p+\mathrm{Au}$ at $2.5 \mathrm{GeV}[11$, 12]. From these analyses, it is found that the leading nucleon with higher kinetic energy, namely higher linear momentum, is unlikely to form any deuterons which are satisfied with the phase space condition given by equation (3) because the relative momentum between the leading nucleon and any other nucleons are large. Consequently, it is expected that the leading nucleon with relatively low kinetic energy is allowed to form LCPs.

\section{Summary and conclusions}

We have proposed a method of improving the QMD prediction of preequilibrium emission of light complex particles in nucleon-induced reactions. A phenomenological surface coalescence model was implemented into the QMD simulation under the assumption that the light complex particles are 

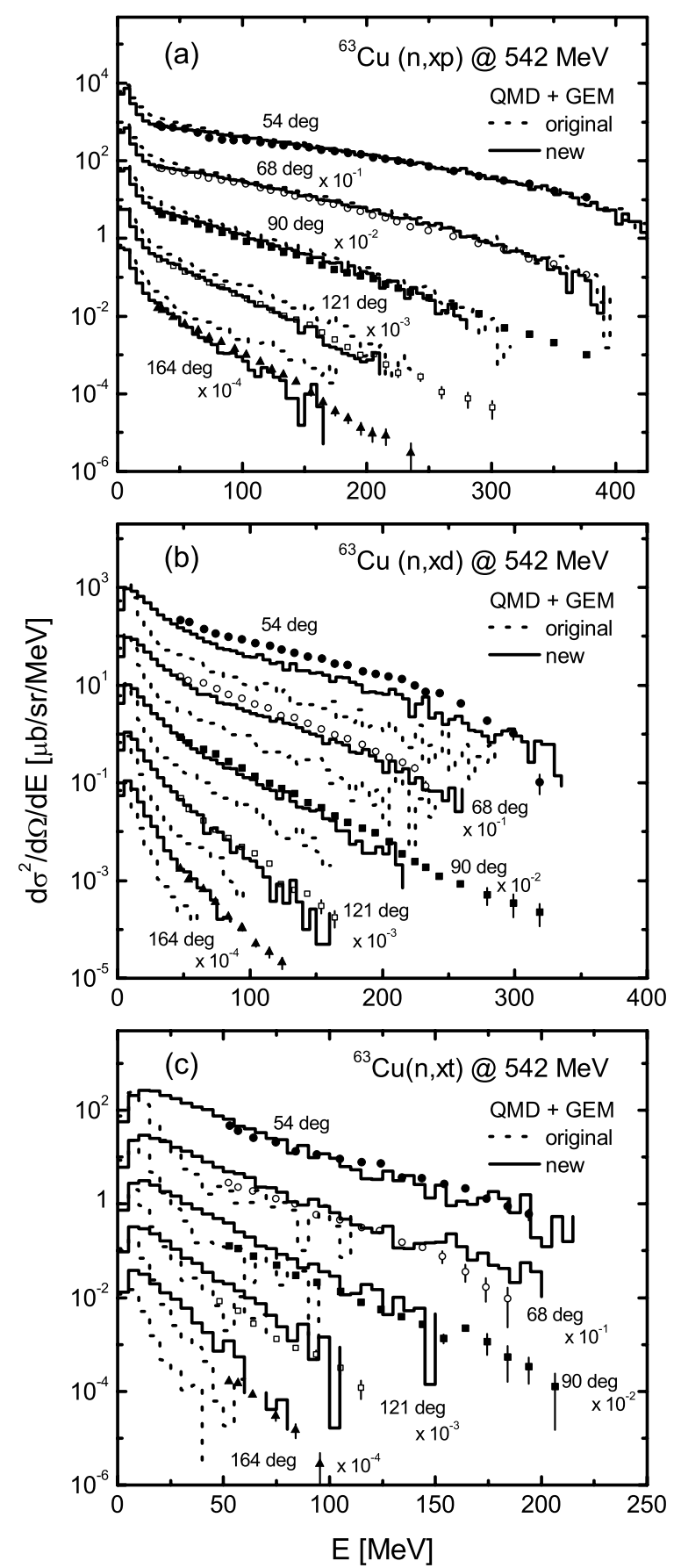

Fig. 3. Double-differential cross sections of hydrogen isotopes produced from the reaction $\mathrm{n}+\mathrm{Cu}$ at $542 \mathrm{MeV}$ : (a) proton, (b) deuteron and (c) triton. The experimental data are taken from ref. [20].

mainly formed near the surface by a leading nucleon that is ready to escape from the nucleus during the dynamical process. The new approach was applied to the neutron-induced reactions on $\mathrm{O}, \mathrm{Si}$, and $\mathrm{Fe}$ at $96 \mathrm{MeV}$ and on $\mathrm{Cu}$ at $542 \mathrm{MeV}$. The result led to the enhancement of the production of light complex particles in the intermediate emission energy range, and the agreement with the experimental data was fairly improved although there still remains underestimation around the high-energy end of these spectra. This discrepancy is possibly related to the direct pick-up process by the incident neutron and the knock-out process of pre-formed clusters which cannot be explained properly in the framework of the coalescence model.

Finally, this work has demonstrated that implementation of the surface coalescence model to the QMD is a promising prescription to improve the production of light complex particles in nucleon-induced reactions. In the future, we aim to apply the extened QMD to further analyses of nucleon-induced lightion production reactions over wide incident energies and target mass numbers in order to find a systematic trend of the model parameters.

The authors would like to thank K. Niita for valuable discussions and encouragement. D.N.K. is grateful to the Japan Society for the Promotion of Science (JSPS) for a postdoctoral fellowship. This work is partly supported by the Grants-in-Aid (No.17-05374) for Scientific Research from JSPS.

\section{References}

1. H.W. Bertini, Phys. Rev. 118, 1711 (1969).

2. A. Boudard et al., Phys. Rev. C 66, 044615 (2002).

3. E. Gadioli, P.E. Hodgson, Pre-Equilibrium Nuclear Reactions (Oxford University Press, New York, 1992), and references therein.

4. J. Aichelin, Phys. Rep. 202, 233 (1991), and references therein.

5. K. Niita et al., Phys. Rev. C 52, 2620 (1995).

6. S. Chiba et al., Phys. Rev. C 54, 285 (1996).

7. Khaled Abdel-Waged, Phys. Rev. C 74, 034601 (2006).

8. Y. Watanabe et al., in Proceedings of the International Conference on Nuclear Data for Science and Technology, Santa Fe, USA, Sept. 26-Oct. 1, edited by R.C. Haight, M.B. Chadwick, T. Kawano, P. Talou (AIP, New York, 2005), p. 326.

9. K. Shibata et al. (these proceedings).

10. H. Iwase et al., J. Nucl. Sci. Technol. 39, 1142 (2002).

11. A. Letourneau et al., Nucl. Phys. 712, 133 (2002).

12. A. Boudard et al., Nucl. Phys. A 740, 195 (2004).

13. A. Iwamoto, K. Harada, Phys. Rev. C 26, 1821 (1982).

14. K. Sato, A. Iwamoto, K. Harada, Phys. Rev. C 28, 1527 (1983).

15. J. Bisplinghoff, Phys. Rev. C 50, 1611 (1994).

16. Y. Watanabe, D.N. Kadrev, in Proceedings of 10th Symposium on Neutron Domimetry, Uppsala, Sweden, June 6-10, 2006, Radiat. Prot. Dosim. (2007) (in press).

17. U. Tippawan et al., Phys. Rev. C 73, 034611 (2006).

18. U. Tippawan et al., Phys. Rev. C 69, 064609 (2004).

19. V. Blideanu et al., Phys. Rev. C 70, 014607 (2004).

20. J. Franz et al., Nucl. Phys. A 510, 774 (1990).

21. T. Maruyama et al., Phys. Rev. C 45, 2355 (1995).

22. S. Furihata, Nucl. Instrum. Meth. Phys. Res. B 171, 251 (2000).

23. M. Garçon, J.W. Van Orden, Adv. Nucl. Phys. 26, 293 (2001). 